\title{
LA-UR-17-27210
}

Approved for public release; distribution is unlimited.

Title:

Author(s):

Intended for:

Issued:
Space Sciences Focus Area

Reeves, Geoffrey D.

Presentation to review committee 
Disclaimer:

Los Alamos National Laboratory, an affirmative action/equal opportunity employer, is operated by the Los Alamos National Security, LLC for the National Nuclear Security Administration of the U.S. Department of Energy under contract DE-AC52-06NA25396. By approving this article, the publisher recognizes that the U.S. Government retains nonexclusive, royalty-free license to publish or reproduce the published form of this contribution, or to allow others to do so, for U.S. Government purposes. Los Alamos National Laboratory requests that the publisher identify this article as work performed under the auspices of the U.S. Department of Energy. Los Alamos National Laboratory strongly supports academic freedom and a researcher's right to publish; as an institution, however, the Laboratory does not endorse the viewpoint of a publication or guarantee its technical correctness. 


\title{
CSES: Center for Space and Earth Science
}
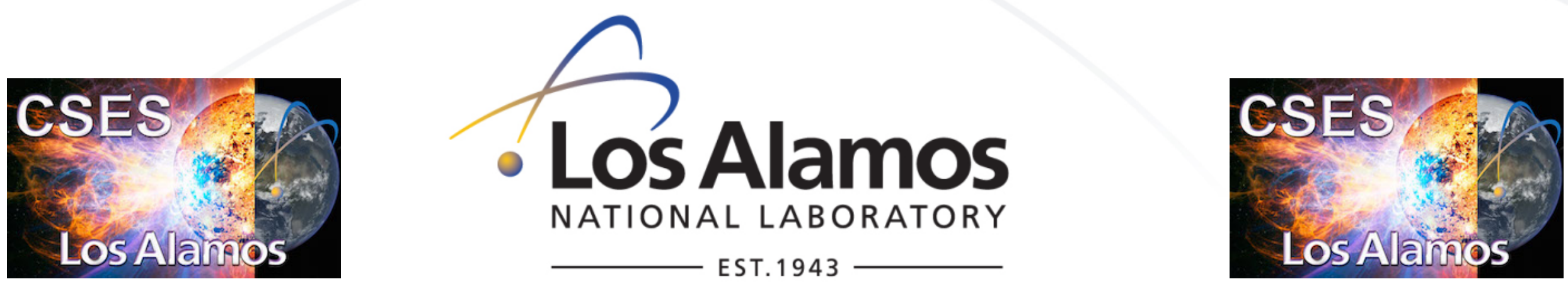

\section{Space Sciences Focus Area}

\author{
Geoff Reeves \\ Space Science and Applications, ISR-1
}

July $18^{\text {th }}, 2017$ 


\section{Space Science Focus Goal}

- To advance our understanding of the space environment (from the Sun to the Earth and beyond) and to advance our ability to operate systems in space that protect life and society. Space Science is distinct from other field, such as astrophysics or cosmology, in that Space Science utilizes in-situ measurements from high altitude rockets, balloons and spacecraft or ground-based measurements of objects and conditions in space. 


\section{Call for proposals}

- We particularly encourage proposals that lead to new missions, new technologies, and/or innovative new uses of unique LANL data, simulation, or modeling capabilities.

- CSES space science focus for this cycle emphasizes research that supports innovation and creativity leading to new missions, new technologies, or new scientific capabilities. 


\section{Call for proposals}

- New space science missions are the "ultimate prize' because they lead to large, sustained research activities, high profile publications, recruitment and training opportunities, etc.

- Often, the path is paved with smaller projects involving the development of new instruments or new measurement capabilities. Those, in turn, often rely on numerical studies that identify the most important open scientific questions and establish the foundational scientific basis. 


\section{New Missions}

- The first priority will be to support LANL's intensifying efforts to develop new NASA (or other) space missions, both for those in the current pipeline

- E.g. CONNEX, IMAP, MMX, Europa Lander, Rocket, Balloon, CubeSat experiments...

- Foundational proposals enabling and/or support mission concept developments for these or new concepts are encouraged. 


\section{New Technologies}

- Technology development and demonstration with an emphasis on technologies that can be applied to basic and national security research objectives:

- Instrument and measurement concepts

- Laboratory demonstrations

- Instrument performance studies that enhance our existing sensors 


\section{New tricks for old capabilities}

- Release of GPS and LANL-Geo data provides new (or renewed) opportunities for collaboration and recruitment. We particularly encourage studies using limited-access data sets that can have open publications (e.g. GPS lightning data). We encourage new numerical model development or use of current model capabilities for completely different applications - particularly those leading to creative and innovative measurement, instrument, and mission concepts. 


\section{Other Opportunities}

- 2018 we will hold the next in the series of very successful LANL Space Weather Summer Schools led by Misa Cowee \& Jesse Woodroffe

- Continue the seminar series Advances in Space Sciences led by Fan Guo \& Jesse Woodroffe

- Sponsor conference organization and conference attendance: e.g. IAGA/IUGG VERSIM workshop, Active Experiments in Space, etc. 